\title{
In-cell infection: bringing uninvited guests
}

\author{
Cell Research (2015) 25:647-648. doi:10.1038/cr.2015.57; published online 8 May 2015
}

Cell-in-cell structures resulting from live cell engulfment were identified more than 100 years ago, but their physiological significance has remained largely obscure. Now Ni et $a l$. identify a new role for cell-in-cell structure formation, called "in-cell infection" that spreads EpsteinBarr virus from infected $B$ cells to epithelial cells, an activity that may predispose to cancer.

Epstein-Barr virus (EBV) is a common herpesvirus infecting up to $90 \%$ or more of the human population that causes mononucleosis, is associated with autoimmune conditions, and predisposes to cancer [1]. EBV persists as a latent infection within $B$ cells and predisposes to cancers of $\mathrm{B}$ cell origin, including Hodgkin's and Burkett's lymphoma, due to expression of latency genes, which leads to B cell transformation. Infected individuals are also predisposed to developing nasopharyngeal and gastric carcinoma, as epithelial cells also harbor latent EBV. However, while the mechanism of EBV entry into B cells is well characterized, how EBV infects epithelium has remained obscure. In a recent paper published in Cell Research, Ni et al. [2] identify a novel mechanism for EBV infection of epithelial cells, which they term "in-cell infection", an insidious mode of viral entry that takes advantage of whole cell ingestion.

Viral infection is generally mediated by viral envelope glycoproteins that bind to specific receptors on target cells, leading to membrane fusion and viral entry. To infect B cells, the EBV envelope protein gp350 binds to the complement receptor 2 (CR2) on target cells, followed by interaction of gp42 with MHC class II molecules, and virus-to-target cell fusion is mediated by gp42, gH and gL proteins [3]. Unlike $\mathrm{B}$ cells, epithelial cells do not normally express complement receptors or MHC class II molecules, and are generally not infected by purified EBV. Previously described alternative modes of EBV infection of epithelial cells include "cell-to-cell" and "transfer" infection, where B cells have been found to act as carriers to mediate infection through cell adhesion protein-dependent conjugation [3-6].

Ni et al. [2] now describe a different mode of epithelial cell infection, called "in-cell infection", that occurs by ingestion of whole EBV-infected B cells, leading to the formation of "cell-in-cell" structures. B cell ingestion in this context resembles "entosis", a mechanism previously found to mediate cell-in-cell structure formation in epithelial cultures and human tumors [7]. Entosis also promotes the uptake of hematopoietic cells into epithelial cells or cancer cells of various types $[8,9]$. Incredibly, the authors find that entosis-like internalization of latent EBV-infected B cells (Akata) into cultured nasopharyngeal carcinoma cells (CNE-2) leads to the activation of EBV and the transfer of virus to host (CNE-2) cells. Cells infected in this manner express viral gene products, and produce virions upon stimulation that can infect naïve cells of either B cell or epithelial cell origin, indicating potent infection ability and altered tropism of EBV produced by this mechanism.

Frequent cell-in-cell structure formation involving EBV-infected B cells is

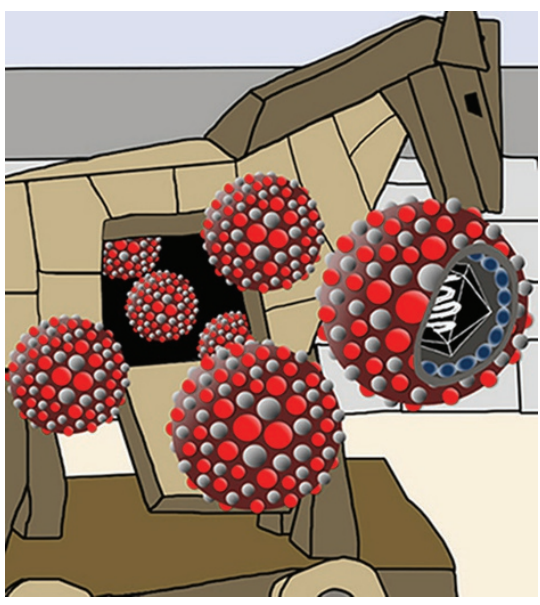

Figure 1 In-cell infection delivers virus to insusceptible host cells. The B cell infected by EBV resembles a Trojan horse that delivers a hidden viral payload to host epithelial cells.

shown by the authors to occur in clinical nasopharyngeal carcinoma samples, suggesting that the in-cell infection mechanism is a likely contributor to viral spread in vivo, and may be linked to carcinoma development. Intriguingly, entosis itself may participate in tumorigenesis by promoting aneuploidy [10], and by supplying cancer cells with nutrients [11]. As the authors found that EBV infection promoted entosis-like cell uptake, this mode of viral spread could affect tumorigenesis by multiple mechanisms. For in-cell infection, it seems that the nutrients taken in upon the death of internalized cells come mixed with virus that is insidiously transferred to hosts, in a manner perhaps like a Trojan horse enterring with a hidden viral payload (Figure 1).

The identification of in-cell infection by Ni et al. [2] makes a significant 
contribution to cell-in-cell research by identifying a new pathophysiological role for an entosis-like process. Cell-incell structures were first reported over 100 years ago, but the mechanisms that control the formation of such structures and their significance are only now starting to emerge [12]. As is often the case with groundbreaking research, the discovery of in-cell infection [2] raises many new interesting questions. What is the mechanism of $\mathrm{B}$ cell internalization into epithelial cells? Entosis is previously described to involve cell adhesion receptors, such as E-cadherin, and Rhokinase that promotes the actomyosin contraction that drives cell uptake [7]. The molecular mechanism controlling the entry of EBV-infected B cells into epithelial cells will be important to un- cover, as other mechanisms in addition to entosis can also mediate the uptake of live cells [12]. How is EBV activated by the formation of cell-in-cell structures? How is EBV transferred from internalized cells to hosts? And importantly, can other viruses, such as HIV, spread by in-cell infection? The answers to these questions await further research.

Yongchan Lee ${ }^{1}$, Michael Overholtzer ${ }^{1}$

${ }^{1}$ Cell Biology Program, Memorial Sloan Kettering Cancer Center, New York, NY 10065, USA Correspondence: Michael Overholtzer

E-mail: overhom1@mskcc.org

\section{References}

1 Niedobitek G, Meru N, Delecluse HJ. Int J
Exp Pathol 2001; 82:149-170.

$2 \mathrm{Ni} \mathrm{C}$, Chen Y, Zeng M, et al. Cell Res 28 April 2015. doi:10.1038/cr.2015.50

3 Shannon-Lowe C, Rowe M. PLoS Pathog 2011; 7:e1001338.

4 Imai S, Nishikawa J, Takada K. J Virol 1998; 72:4371-4378.

5 Shannon-Lowe CD, Neuhierl B, Baldwin G, et al. Proc Natl Acad Sci USA 2006; 103:7065-7070.

6 Nanbo A, Terada H, Kachi K, et al. J Virol 2012; 86:9285-9296.

7 Overholtzer M, Mailleux AA, Mouneimne G, et al. Cell 2007; 131:966-979.

8 Wang S, He MF, Chen YH, et al. Cell Death Dis 2013; 4:e856.

9 Wang S, Guo Z, Xia P, et al. Cell Res 2009; 19:1350-1362.

10 Krajcovic M, Johnson NB, Sun Q, et al. Nat Cell Biol 2011; 13:324-330.

11 Krajcovic M, Krishna S, Akkari L, et al. Mol Biol Cell 2013; 24:3736-3745.

12 Overholtzer M, Brugge JS. Nat Rev Mol Cell Biol 2008; 9:796-809. 